\title{
How to measure endoscopy unit performance: some metrics for dummies
}

Author

Institution
V. Raman Muthusamy

University of California, Los Angeles - Medicine/Digestive Diseases, Los Angeles, California, United States submitted 19. January 2016 accepted after revision 29. January 2016

\section{Bibliography}

Dol http://dx.doi.org/ 10.1055/s-0042-102249 Endoscopy International Open 2016; 04: E149-E150 (c) Georg Thieme Verlag KG Stuttgart $\cdot$ New York E-ISSN 2196-9736

Corresponding author

\section{Raman Muthusamy, MD,} FACG, FASGE

University of California, Los Angeles - Medicine/Digestive Diseases

200 UCLA Medical Plaza Room 330-37

Los Angeles CA 90095

Phone: +1-310-825-1892

Fax: $+1=310-825-5429$

raman@mednet.ucla.edu
As the diagnostic and therapeutic indications for endoscopy continue to expand, the demand for these procedures has steadily increased. This demand has been compounded by increases in the number of patients with access to care as well as an aging and enlarging population. These developments have led to significant challenges regarding endoscopic access, often due to limitations in physical space or personnel. Despite the importance of optimizing endoscopy unit efficiency, however, limited studies have been conducted in this area. Among the many challenges faced in performing such research is what variables or metrics to measure, exactly how to measure them, and how many to study. Some background regarding metrics may facilitate an understanding of their role in assessing efficiency and quality.

Metrics are measurements specifically designed to assess some quantifiable component of an organization's performance [1]. Metrics can be developed with respective to the stakeholder (endoscopist, facility, payor, or patient) or by using the conceptual framework described by Donabedian in which operational performance is grouped into structure, process, and outcome categories [2,3]. Structural metrics include unit size and layout, the number of endoscopists and staff, and the preferred method of sedation. Process metrics include first case start time and preparation, sedation, procedure, recovery, and room turnover times. Outcomes metrics measure output such as unit or room procedural throughput, costs, resource use, and patient wait times. The Agency for Healthcare Research and Quality has identified foiur important components for proposed efficiency metrics [4]. They must: 1) possess relevance/significance and be under of the control of one of the aforementioned stakeholders; 2 ) be reliable, reproducible, and scientifically valid as a measure of efficiency; 3 ) be feasible to measure; and 4) be actionable to allow for the po- tential of improvement. Benchmarks can be established once metrics from a large number of units are available, and allow a facility to assess its value/success in comparison to similar units. Traditional research in endoscopy unit efficiency has focused on the effect of process metrics, such as room turnover time and first case start time, on outcome metrics. This is likely due to the fact that changes in processes are more feasible than structural modifications, which are resource intensive. Variability in metric definitions, such as what constitutes "late" and the start and stop times of various intervals, is common among studies. In addition to the traditional process metrics, the "true completion time" (TCT) has been recently proposed as a metric to better assess the impact of delays early in the day on unit operations and patient waiting times [5]. The TCT is defined as the time from the scheduled procedure start time to the time the patient exits the room postprocedure. It is meant to capture the effect of a single delayed procedure early in the day on patient-experienced delays throughout the day, even if all other cases are performed within the expected duration. Despite the large number of potential metrics, the exact combination of metrics that will best characterize a unit's efficiency remain unclear and will likely vary based on specific unit characteristics.

Prior research in this field has traditionally used a "time-and-motion" methodology and has been assessed from the perspective of the facility or of the endoscopist [6]. An initial study from an endoscopist's perspective found that reducing the room turnover time between procedures was associated with improved efficiency [7]. Other potentially beneficial factors included either using an individual other than the endoscopist to provide sedation or having two rooms per endoscopist. Factors associated with inefficiency have included physician unavailability, inadequate space in the pre-procedure/recovery area, and in- 
efficient scheduling $[5,8,9]$. In many cases, effective solutions can be found that require minimal, if any, increase in cost. For example, many solutions involve performing several tasks simultaneously rather than sequentially $[10,11]$. Other simple but successful strategies have including bringing the first patient of the day directly into the procedure room to save pre-procedure/recovery room space and altering the timing and duration of appointments $[5,9]$.

In this issue, Yang and colleagues provide a rare glimpse into the operations of an interventional endoscopy unit [12]. Specifically, efficiency metrics were collected over 6 months from two interventional endoscopy rooms in a four-room academic hospitalbased endoscopy unit treating both outpatients and inpatients. On-time room starts occurred within 15 minutes of the scheduled time in $85 \%$ of cases, with a mean procedure time of 27.5 minutes, and a mean patient in-room time of 52.1 minutes. However, the mean time from patient exit to subsequent patient entry was 37.1 minutes. About half of the non-endoscopy time was due to delays in patient processing. In total, non-endoscopy time comprised two-thirds of the total room time, leading to less than five procedures being performed per room per day. It is interesting that on-time room starts were organizationally defined as having the patient in the room by the procedure start time but room turnover time was not considered delayed unless more than 30 minutes had elapsed. These data show that the focus on on-time room starts (a traditional operating room metric in which delays are often due to physician unavailability) may not be the primary factor limiting efficiency when a high volume of cases are performed per room daily, as in endoscopy units. In this case, it appears that the practice of inserting inpatients (one-third of study population) throughout the day's schedule led to many delays due to patient processing and transport.

The authors acknowledge the major limitation of their study, the lack of an intervention phase designed to implement process modifications to assess if unit efficiency was enhanced. Given the study findings, potential solutions suggested in their discussion included only doing inpatients after all outpatient procedures were completed and obtaining all inpatient anesthesia/ endoscopy procedure consents and completing their pre-procedure assessments prior to arrival in the endoscopy unit. A follow-up study reporting the effects of these proposed interventions would be instructive.

Optimizing efficiency in endoscopy units remains a challenging process given the many variables involved. The key bottlenecks to efficiency will vary based on variables such as physical space, staffing, method and personnel involved with sedation, procedure mix, and operational procedures specific to each unit. However, recognizing the need to track patient flow through the endoscopy unit is the common first step in improving efficiency. This information should then be analyzed for potential interventions, and when possible, simulations of the proposed changes should be performed to identify the optimal strategy [9]. These modifications should then be implemented to determine their ef- fect on key metrics. Additional steps not involving the assessment of specific metrics include developing a culture of collaboration and incentivizing endoscopists, anesthesia providers, and endoscopy unit staff to achieve pre-specified quality and productivity goals.

As electronic health records are more widely adopted, acquiring data regarding key process measures such as those recorded by Yang and colleagues should become more feasible. The incorporation of this information into yet-to-be-developed large-scale national databases containing endoscopic operational metrics has the potential to provide a more complete picture of endoscopy unit operations. Ultimately, such an effort could allow for the development of valid, widely agreed upon metrics to assess endoscopy unit performance and the establishment of benchmarks for endoscopic efficiency.

In summary, the authors of this study should be commended for sharing the findings of the operational data from their unit. In an era of declining procedural reimbursement, continued research into endoscopy unit efficiency is essential to reduce costs while maintaining and hopefully maximizing quality. Most importantly, from a patient perspective, such work has the potential to aid in enhancing satisfaction associated with receiving endoscopic procedures while improving access to them.

\section{Competing interests: None}

\section{References}

1 Day LW, Belson D. Studying and Incorporating Efficiency into Gastrointestnal Endoscopy Centers. Gastroenterol Res Pract 2015; 2015: 764153

2 Donabedian A. The quality of care. How can it be assessed? JAMA 1988; 260: $1743-1748$

3 Gellad ZF, Thompson CP, Taheri J. Endoscopy unit efficiency: quality redefined. Clin Gastroenterol Hepatol 2013; 11: 1046-1049 e1

4 Gellad ZF. Endoscopy unit efficiency. Gastroenterol Hepatol (N Y) 2014; 10: 315-317

5 Kaushal NK, Chang K, Lee JG et al. Using efficiency analysis and targeted intervention to improve operational performance and achieve cost savings in the endoscopy center. Gastrointest Endosc 2014; 79: 637645

6 Harewood GC, Chrysostomou K, Himy N et al. A "time-and-motion" study of endoscopic practice: strategies to enhance efficiency. Gastrointest Endosc 2008; 68: 1043-1050

7 Zamir S, Rex DK. An initial investigation of efficiency in endoscopy delivery. Am J Gastroenterol 2002; 97: 1968-1972

8 Yong E, Zenkova O, Saibil $F$ et al. Efficiency of an endoscopy suite in a teaching hospital: delays, prolonged procedures, and hospital waiting times. Gastrointest Endosc 2006; 64: 760-764

9 Day LW, Belson D, Dessouky $M$ et al. Optimizing efficiency and operations at a California safety-net endoscopy center: a modeling and simulation approach. Gastrointest Endosc 2014; 80: 762 - 773

10 Olmstead J, Coxon P, Falcone D et al. World-class OR turnaround times: secrets uncovered. AORN J 2007; 85: 942-945, 947-949

11 Friedman DM, Sokal SM, Chang $Y$ et al. Increasing operating room efficiency through parallel processing. Ann Surg 2006; 243: 10-14

12 Yang $D$, Summerlee $R$, Suarez $A$ et al. Evaluation of interventional endoscopy unit efficiency metrics at a tertiary care academic medical center. Endoscopy International Open 2016: 4 